\title{
Opioid Use in the Postpartum Period: Are We Prescribing Too Much?
}

Danielle Prentice, DO; Amanda Berry, DO; Linzi Stewart, DO; Heather Wilkins, PhD; Serdar Ural, MD; Raymond Deiter, DO

From the Department of Obstetrics and Gynecology at

St. Anthony Hospital in Oklahoma City, Oklahoma (Drs Prentice, Berry, Stewart,

and Deiter); Integris Southwest Medical Center in Oklahoma City, Oklahoma (Drs Prentice, Hill, Stewart,

and Deiter); Penn State Health Milton S. Hershey Medical Center in Hershey, Pennsylvania (Drs Prentice and Ural); and University of Kansas Medical Center in Kansas City (Dr Wilkins).

Financial Disclosures:

None reported.

Support: None reported.

Address correspondence to Danielle Prentice, DO, Penn

State Milton S. Hershey Medical Center, 500 University Blvd, Hershey, PA 17033-2360.

Email:

dprentice@pennstatehealth psu.edu

Submitted August 13, 2019; revision received October 25, 2019; accepted November 11, 2019
Context: The first exposure to opioids for many women of reproductive age follows childbirth. Current data show a significant correlation between the number of days supplied and continued use/abuse of opioids. The number of women with opioid dependency in pregnancy is steadily increasing, and opioid use is directly linked to an increase in maternal and neonatal morbidity and mortality. However, there are no clear opioid-prescribing guidelines for the postpartum period.

Objective: To compare the number of opioid pills prescribed with the number used by patients in the postpartum period.

Methods: Patients were recruited to this pilot study at the time of admission to the labor and delivery unit at a community hospital in Oklahoma City, Oklahoma; 84 patients gave informed consent to participate. Medical records were reviewed to determine the number of opioids prescribed. Phone surveys were conducted 4 to 6 weeks after discharge to identify the number of opioids used during the postpartum period. Welch $t$ test was used to determine $P$ values.

Results: After exclusion criteria were applied, records of 23 patients with vaginal deliveries and 14 patients with cesarean sections were included in the study. Patients who were prescribed opioids after a vaginal delivery were prescribed significantly more pills than were used $(P<.001)$; a mean of 10 opioid pills per patient remained unused. Patients prescribed opioids after cesarean section were also often prescribed more opioid pills than used $(P<.05)$; a mean of 7.5 opioid pills per patient remained unused. Of 37 patients, only 2 disposed of unused opioid pills.

Conclusion: The data in this study show a clear example of overprescribing opioids after vaginal and cesarean deliveries leading to increased opioid pill availability within the community.

J Am Osteopath Assoc. 2020;120(10):698-703. Published online August 5, 2020

doi:10.7556/jaoa.2020.114

Keywords: narcotics, obstetrics, opiates, opioid abuse, postpartum

$\mathrm{T}$ he US is currently in the midst of an opioid epidemic. More than 11.4 million people misuse prescription opioids. ${ }^{1,2}$ About 2.1 million of these people have an opioid-use disorder. ${ }^{1}$ It is estimated that 130 people die daily from opioid-related overdose deaths, which amounts to over 47,000 deaths per year. ${ }^{2}$ The first exposure to opioids for many women of reproductive age is at childbirth. ${ }^{3}$ Current data show a significant correlation between the number of days for which opioids are prescribed and patients' continued use of opioids. For example, a 5-day supply is linked to 
a $10 \%$ chance of continued use at 1 year and a $5 \%$ chance of continued use at 3 years. ${ }^{4,5}$ The risk of persistent opioid use after the postpartum period is associated with a single prescription, regardless of drug delivery route. ${ }^{3}$

In a 2019 US national cohort study, ${ }^{6}$ women who received a peripartum opioid prescription had new persistent opioid use rates of 1.7 and $2.2 \%$ for vaginal births and cesarean sections, respectively. Furthermore, 1 in 300 women who were prescribed opioids after a cesarean section became opioid-dependent. ${ }^{7}$ The number of women with opioid dependency in pregnancy is steadily increasing, and more women are being treated for this dependency during pregnancy. ${ }^{8}$ The result of this increase in opioid dependency in women is an increased rate of neonatal abstinence syndrome. Opioid use is directly linked to an increase in maternal and neonatal morbidity and mortality, which have been on the rise in the US. ${ }^{9,10}$ However, there are no clear opioid-prescribing guidelines for the postpartum period.

Oklahoma has historically had 1 of the highest rates of opioid prescriptions, at 104.4 per 100 people in 2015. ${ }^{11}$ Through significant efforts by the Oklahoma Department of Health, the Oklahoma legislature, and physicians, this rate improved to 88.1 opioid prescriptions per 100 people in 2017. ${ }^{11}$ However, more improvement is imperative. Of the 3533 overdose deaths in Oklahoma between 2011 and 2015, 2113 were attributed to the misuse of prescription opioids. ${ }^{11}$ Importantly, 9 out of 10 overdose deaths in Oklahoma related to opioids were unintentional. ${ }^{11}$

Oklahoma has an established maternal mortality review committee that analyzes factors involved with maternal deaths. Between 2004 and 2016, 8.7\% of maternal deaths in Oklahoma were deemed opiate-related. Of these deaths, $25 \%$ occurred during pregnancy and $75 \%$ during the postpartum period. ${ }^{11}$ Recent state legislation with the passing of Oklahoma House Bill 2334 established a more comprehensive maternal mortality committee to facilitate accurate data collection and analysis. ${ }^{12}$
There is evidence in the literature that many opioid prescription pills are not used after surgical procedures. A systematic review by Bicket et $\mathrm{al}^{13}$ found that $41 \%$ to $72 \%$ of opioid pills were unused following several different surgical procedures. Included in the aforementioned systematic review is a study that observed 30 cesarean section patients in the postoperative period; about $83 \%$ of these women took less than half of the opioids prescribed. ${ }^{14}$ Using data from vaginal and cesarean section delivery patients in our institution, we sought to assess whether women were overprescribed opioids in the postpartum period, which may lead to misuse, abuse, and a dangerous number of unused opioid pills entering the community.

\section{Methods}

This prospective, cross-sectional pilot study was performed at a community hospital in Oklahoma City, Oklahoma, between March 1, 2018 and October 31, 2018. The study was approved by the Integris Internal Review Board (IRB+ 18-014, 2018). The study concluded early because of a change in Oklahoma opioidprescribing laws with Oklahoma Senate Bill that went into effect on November 1, 2018, and had the potential to introduce bias. The new law mandated that the maximum amount of opioids that could be prescribed was a 7-day supply and that this supply could be replenished via an in-person visit twice. ${ }^{15}$ Furthermore, the law introduced a consenting protocol that included the possible risks of dependence, overdose, and death.

Patients were recruited to the study on admission to the labor and delivery unit at our institution. Written informed consent was obtained by obstetrics and gynecology residents covering the unit. Medical record reviews were performed to determine the delivery method and type and number of opioids prescribed. Phone surveys were performed 4 to 6 weeks after delivery to determine the number of opioid pills used. A secondary question was included to determine whether and how patients were disposing of their unused medication. If the phone call was not answered, a detailed 
voicemail was left. A total of 3 phone call attempts were made. Inclusion criteria were age 18 to 45 years, delivery at Integris Southwest Medical Center during the study period, prescription of opioids by the attending physician upon discharge from the labor and delivery unit, institution-standard opiate prescriptions of hydrocodone-acetaminophen 5-325 $\mathrm{mg}$ for vaginal delivery or oxycodone-acetaminophen 5-325 mg for cesarean delivery, and a working home or cell phone number to be contacted 4 to 6 weeks postpartum. This inclusion criterion was a recommendation by the IRB for standardization of patients in the study. Exclusion criteria included any of the above inclusion criteria not being met; inability to reach patient by phone in the postpartum period; current care under pain management physician or use of buprenorphine, buprenorphine/naloxone, or methadone; and history of opioid addiction, including heroin or prescription pain medications. The mean was calculated for the number of opioid pills prescribed with vaginal delivery and cesarean section, respectively, and the number of opioid pills used in each category. An F test was performed which showed the standard deviation to be significantly different between groups. Therefore, Welch $t$ test, which controls for unequal variance between groups, was used to determine statistical significance.

\section{Results}

A total of 84 women gave informed consent for this study upon arrival to the labor and delivery unit. Of these 84 women, 14 (17\%) were excluded due to receiving a prescription for an opioid other than those listed in the inclusion criteria. Furthermore, 33 patients (39\%) were unable to be contacted in the 4 to 6 week postpartum period. Statistical analysis included 23 patients $(27 \%)$ with vaginal deliveries and 14 patients (17\%) with cesarean sections.

The mean (SD) number of hydrocodone-acetaminophen 5-325 mg prescribed for vaginal deliveries was 18.5 (4.2) pills (range, 12 to 30), and the mean taken was 7.8 (8.2) pills. (Table) Patients prescribed opioids

Table.
Number of Opioid Pills Prescribed and Used
Following Vaginal and Cesarean Section Delivery
at a Community Hospital in Oklahoma City,
Oklahoma
\begin{tabular}{cccc} 
Delivery type & Mean (SD) & Range & $\mathbf{9 5 \%}$ Cl \\
\hline Vaginal deliveries & & & \\
\hline Prescribed & $18.5(4.2)$ & 20 & $20.4-16.7$ \\
\hline Used & $7.8(8.2)$ & 20 & $11.4-4.3$ \\
\hline Cesarean section & & & \\
\hline Prescribed & $22.1(4.3)$ & 10 & $24.6-19.7$ \\
\hline Used & $14.6(9)$ & 30 & $19.8-9.5$ \\
\hline
\end{tabular}

Abbreviations: $\mathrm{Cl}$, confidence interval; $\mathrm{SD}$, standard deviation

after a vaginal delivery were prescribed significantly more opioid pills than used $(P<.001$; Figure 1); a mean of 10.7 opioid pills per patient went unused (Table).

The mean (SD) number of oxycodone-acetaminophen 5-325 mg prescribed for cesarean sections was 22.1 (4.3) pills (range, 20 to 30 pills), and the mean number taken was 14.6 (9) pills. (Table) Patients prescribed opioids after cesarean section were also often prescribed more opioids than used $(P<.05$; Figure 1); a mean of 7.5 opioid pills per person were unused (Table).

The mean number of unused opioids across the study population was 9.1 pills. Of the 37 patients, only 2 (5\%) disposed of unused opioid pills. No statistical significance was seen for differences in opioid use based on age, race/ethnicity, or parity. Based off medical record review, this was also true for those who were unable to be reached for the survey.

\section{Discussion}

Our prospective, cross-sectional pilot study found that opioids were being prescribed at our study site in the postpartum period at much higher rates than they were being consumed. We demonstrated that the association was present for both vaginal deliveries and cesarean sections. The purpose of this study was to establish 

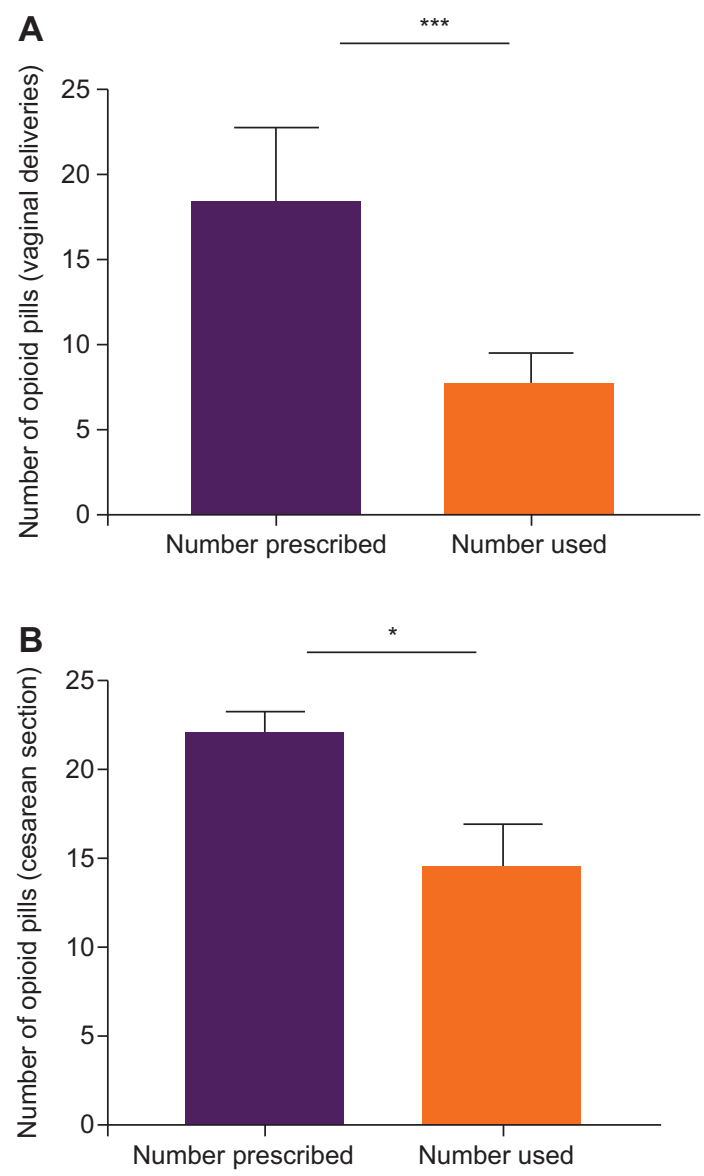

Figure.

Opioid pills prescribed and used following $(A)$ vaginal delivery ( $P<.001$ via $t$ test with Welch correction), and $(\mathrm{B})$ cesarean section ( $P=.01$ via $t$ test with Welch correction). ${ }^{* * *} P<.0001 ;{ }^{*} P<.05$.

data to better guide opioid-prescribing guidelines in the postpartum period.

As noted previously, the study was concluded early due to a change in Oklahoma opioid prescribing laws effective November 1, 2018, that had the potential to introduce uncontrolled bias. The new law mandated that the maximum number of opioids that could be prescribed was a 7-day supply, and this supply could be replenished via an in-person visit twice. Furthermore, the law introduced a consenting protocol that discussed the possible risks of dependence, overdose, and death. Although this protocol did not necessarily change pre- scribing by providers, the increased education could possibly change patient behavior or reporting on the number of opioids used. Due to this possibility and the inability to control for it, the study was concluded early.

Our study site averages approximately 1200 deliveries per year. If each of these women was given a prescription for opioids at the rates prescribed during this study, 10,920 unused pills (based on our mean of 9.1 unused pills across the study population) would enter the community each year. Historically, Oklahoma has led the nation in misuse and abuse of opioids, which makes this number noteworthy. Furthermore, these striking results are from a single department at 1 hospital. Oklahoma has made large strides in curtailing prescription opioid deaths. However, in 2017, more than 251 people in Oklahoma died due to unintentional overdose from the misuse of opioids prescribed by a physician. ${ }^{11}$ Overall, if prescribing habits change based on the results of this study in all departments and hospitals, the number of opioid pills entering the community would be significantly reduced.

It is evident that, based on these numbers, standard guidelines for prescribing in the postpartum period should lower the number of opioid pills entering the community, as well as lower the potential for misuse and abuse. Following the conclusion of our study and based on its results as presented to the department in an author's (D.P.) resident project, prescribing practices have become more standard at our institution. Patients with uncomplicated vaginal deliveries are not prescribed opioids, which is a significant change at our study site. If a patient has a complicated vaginal delivery or vaginal lacerations and desires an opioid prescription with current inpatient, she is typically prescribed 10 hydrocodone-acetaminophen 5-325 mg. Cesarean sections are now typically prescribed 20 oxycodone-acetaminophen 5-325 mg with current inpatient use.

The secondary question asked of patients in this study was, "How did you dispose of your unused opioids?" Only 2 patients disposed of them. One "flushed them" and the other used a disposal program at her pediatrician's office. Disposal programs are 
widespread in Oklahoma at various hospitals, doctors' offices, and pharmacies. The Oklahoma Bureau of Narcotics and Dangerous Drugs Control coordinates the program Safe Trips for Scripts. ${ }^{16}$ These programs are underused, likely because the education and marketing of these programs are lacking. Increasing use of disposal programs would help to curtail the number of unused opioids entering the community.

The strength of this study is prospective insight into opiate prescribing trends in a patient population that is particularly vulnerable to first-time opioid exposure and continued opioid use. The lack of standardization in prescribing was evident throughout this study. More opioids than required were uniformly prescribed. This is clearly important in a state that has a high rate of opioid prescriptions and a large number of unused opioids entering the community. ${ }^{17}$

The main limitation in this study is sample size. Although the study was able to show statistical significance, a larger sample size would strengthen our conclusions. The sample size was smaller than anticipated due to multiple factors, which included the need to conclude the study early due to Oklahoma opioid prescribing law changes, difficulty contacting patients at the 4 to 6 week postpartum follow-up phone call, and tracking opioids prescribed at a rate or type other than at the standard guidelines set in the inclusion criteria. Possible biases that can be attributed to this study include selection and recall bias. Patients who decided to participate in the study were possibly more likely to feel like they would not have a problem with misusing or abusing opioids. Furthermore, self-reporting and monitoring of the number of pills taken could lead to misreporting. Additionally, there is also the possibility that those who were unable to be reached by phone would have significantly changed the results of this study.

In future studies, morphine equivalents could be used instead of specific formulations of opioid medications to include more participants. Furthermore, the study data collection period should be lengthened, with multiple sites included. Follow-up with patients could occur at the 6-week in-person postpartum visit to reduce dropout at the follow-up phone call. Current research at the same institution is focusing on disposalmethod education to decrease the number of opioids entering the community. This ongoing study is using the pilot study reported here as a baseline for rates of disposal, as well as for aiding in statistical power calculations, recruitment, and dropout rates. Randomized clinical trials are now being performed on ibuprofen and acetaminophen as scheduled pain relief medications added to the standard pain regimens used in this study to decrease the overall use of opioid medications. Programs for opioid-prescribing consents, pain contracts, and patient education have been instituted; the effects of these initiatives have not yet been observed. Initiatives such as these should be a focus of future studies.

\section{Conclusion}

The data in this study show a clear example of overprescribing opioids after vaginal and cesarean deliveries leading to increased opioid pill availability within the community.

\section{Author Contributions}

All authors provided substantial contributions to conception and design, acquisition of data, or analysis and interpretation of data; all authors drafted the article or revised it critically for important intellectual content; all authors gave final approval of the version of the article to be published; and all authors agree to be accountable for all aspects of the work in ensuring that questions related to the accuracy or integrity of any part of the work are appropriately investigated and resolved.

\section{References}

1. Key Substance Use and Mental Health Indicators in the United States: Results from the 2017 National Survey on Drug Use and Health. HHS Publication No. SMA 18-5068. Substance Abuse and Mental Health Services Administration/US Department of Health and Human Services; 2018.

2. Rudd RA, Aleshire N, Zibbell JE, Gladden RM. Increases in drug and opioid overdose deaths - United States, 2000-2014. MMWR Morb Mortal Wkly Rep. 2016;64(50):1378-1382. https://www.cdc.gov/mmwr/ preview/mmwrhtml/mm6450a3.htm. Accessed July 29, 2020.

3. Osmundson SS, Wiese AD, Min JY, et al. Delivery type, opioid prescribing, and the risk of persistent opioid use after delivery. $A m$ 
J Obstet Gynecol. 2019;220(4):405-407. doi:10.1016/j. ajog.2018.10.026

4. Mojtabai R. National trends in long-term use of prescription opioids. Pharmacoepidemiol Drug Saf. 2018;27:526-534. doi:10.1002/ pds. 4278

5. Shah A, Hayes CJ, Martin BC. Characteristics of initial prescription episodes and likelihood of long-term opioid use-United States, 20062015. MMWR Morb Mortal Wkly Rep. 2017;66(10):265-269. doi:10.15585/mmwr.mm6610a1

6. Peahl AF, Dalton VK, Montgomery JR, Lai YL, Hu HM, Waljee JF. Rates of new persistent opioid use after vaginal or cesarean birth among US women. JAMA Netw Open. 2019;2(7):e197863. doi:10.1001/jamanetworkopen.2019.7863

7. Bateman BT, Franklin JM, Bykov K, et al. Persistent opioid use following cesarean delivery: patterns and predictors among opioid-naïve women. Am J Obstet Gynecol. 2016;215(3):353.e1-353. e18. doi:10.1016/j.ajog.2016.03.016

8. Haight SC, Ko JY, Tong VT, Bohm MK, Callaghan WM. Opioid use disorder documented at delivery hospitalization-United States, 1999-2014. MMWR Morb Mortal Wkly Rep. 2018;67(31):845-849. doi:10.15585/mmwr.mm6731a1

9. Corr TE, Hollenbeak CS. The economic burden of neonatal abstinence syndrome in the United States. Addiction. 2017;112(9):1590-1599. doi:10.1111/add.13842

10. Winkelman TNA, Villapiano N, Kozhimannil KB, Davis MM, Patrick SW. Incidence and costs of neonatal abstinence syndrome among infants with Medicaid: 2004-2014. Pediatrics. 2018;141(4):e20173520. doi:10.1542/peds.2017-3520
11. Oklahoma: Opioid-Involved Deaths and Related Harms. National Institute on Drug Abuse; 2020. https://www.drugabuse.gov/drug-topics/ opioids/opioid-summaries-by-state/oklahoma-opioid-involved-deathsrelated-harms. Accessed July 19, 2020.

12. Maternal Mortality Review Act. HR 2334, 57th Leg, 1st Sess (OK 2019). Available at: www.oklegislature.gov/Billlnfo.aspx? Bill=HB2334. Accessed June 29, 2020.

13. Bicket MC, Long JJ, Pronovost PJ, Alexander GC, Wu CL. Prescription opioid analgesics commonly unused after surgery: a systematic review. JAMA Surg. 2017;152(11):1066-1071. doi:10.1001/ jamasurg.2017.0831

14. Bartels K, Mayes LM, Dingmann C, Bullard KJ, Hopfer CJ, Binswange IA. Opioid use and storage patterns by patients after hospital discharge following surgery. PLoS One. 2016;11(1):e0147972. doi:10.1371/journal.pone.0147972

15. S 1446, 57th Leg, 2nd Sess (OK 2020). Available at: www. oklegislature.gov/BillInfo.aspx?Bill=sb1446. Accessed June 29, 2020.

16. Safe use, storage, and disposal. Oklahoma State Department of Health website. www.ok.gov/health/Prevention_and_Preparedness/ Injury_Prevention_Service/Drug_Overdose/Safe_Use,_Storage_and_ Disposal/index.html. Accessed July 19, 2020.

17. Oklahoma opioid prescribing guidelines. Oklahoma Hospital Association website. www.okoha.com/OHA/Health_Care_Issues/ Quality_Patient_Safety/Oklahoma_Opiod_Guidelines/OHA/Health_ Care_Issues/Patient_Safety/Opioid_Prescribing_Guidelines/ Oklahoma_Opioid_Guidelines.aspx?hkey=ab7ed086-9413-40cbb165-3cecfc055a07. Accessed July 29, 2020.

๑ 2020 American Osteopathic Association 